\title{
Mineração
}

\section{Metodologia para análise da confiabilidade estrutural de escavações em rocha}

\section{(Methodology for the analysis of structural reliability in rock excavations)}

\author{
Eduardo César Sansone \\ Departamento de Engenharia de Minas e de Petróleo da Escola Politécnica da Universidade de São Paulo \\ E-mail:esansone@usp.br \\ Luiz Carlos Rusilo \\ Pontifícia Universidade Católica de São Paulo \\ E-mail: Icrusilo@gmail.com \\ Lineu Azuaga Ayres da Silva
}

Departamento de Engenharia de Minas e de Petróleo da Escola Politécnica da Universidade de São Paulo

E-mail: layres@usp.br

\section{Resumo}

Esse artigo apresenta uma metodologia para incorporação de incertezas à modelagem numérica pelo método dos elementos distintos em análises de confiabilidade estrutural de escavações em rocha. A metodologia foi desenvolvida levando em conta que os métodos de modelagem numérica normalmente empregados fornecem resultados pontuais, determinados a partir de parâmetros de entrada fixos, sem que sejam considerados seus respectivos erros. Entretanto a análise da estabilidade de estruturas em rocha envolve incertezas decorrentes da variabilidade inerente aos processos geológicos, da incerteza a respeito da hipótese de comportamento mecânico adotada e, também, das incertezas associadas aos parâmetros adotados na formulação do modelo numérico. Essas fontes de incerteza podem ser levadas em conta em modelos determinísticos simples, mas, na modelagem numérica, onde os resultados são de diversas naturezas, cabendo ao pesquisador analisá-los para obter suas conclusões, faz-se necessário o desenvolvimento de uma metodologia nova, baseada em simulações de Monte Carlo, com o emprego dos princípios da Lógica Paraconsistente, e que será apresentada na análise de um talude final de uma cava de mineração de grandes dimensões.

Palavras-chave: Modelos numéricos, método dos elementos distintos, Lógica Paraconsistente, mineração, talude.

\begin{abstract}
A methodology for rock-excavation structuralreliability analysis that uses Distinct Element Method numerical models is presented. The methodology solves the problem of the conventional numerical models that supply only punctual results and use fixed input parameters, without considering its statistical errors. The analysis of rock-excavation stability must consider uncertainties from geological variability, from uncertainty in the choice of mechanical behaviour hypothesis, and from uncertainties in parameters adopted in numerical model construction. These uncertainties can be analyzed in simple deterministic models, but a new methodology was developed for numerical models with results of several natures. The methodology is based on Monte Carlo simulations and uses principles of Paraconsistent Logic. It will be presented in the analysis of a final slope of a largedimensioned surface mine.
\end{abstract}

Keywords: Numerical models, distinct element method, paraconsistent logic, mining, slope. 


\section{Introdução}

O engenheiro de minas deve projetar estruturas subterrâneas, como túneis e galerias, ou a céu aberto, como por exemplo, taludes. Outras vezes, confronta-se com a necessidade de estudar estruturas anteriormente construídas, ou mesmo, estruturas naturais como as encostas. Em todos esses casos, o engenheiro deve verificar se a estrutura atende aos padrões de segurança exigidos e, portando, precisa avaliar sua condição de estabilidade, podendo usar vários métodos de análise, como as formulações empíricas, os equacionamentos analíticos e a modelagem numérica (Sansone, 1997).

Entre esses métodos, a modelagem numérica é uma ferramenta que vem sendo utilizada cada vez mais intensamente no projeto, análise e decisão, dispondose de diversos tipos, como o método dos elementos finitos, o método dos elementos de fronteira, o método dos elementos distintos e outros. Entretanto os métodos normalmente utilizados são fundamentalmente determinísticos, ou seja, fornecem resultados que são calculados a partir de parâmetros de entrada definidos de forma rigorosa, sem que sejam considerados seus respectivos erros (Jing, 2003).

A avaliação da estabilidade de estruturas em rocha, por outro lado, envolve incertezas decorrentes de fatores como a variabilidade inerente aos processos naturais, a incerteza sobre a hipótese de comportamento adotada e as incertezas associadas aos parâmetros geométricos e mecânicos do modelo numérico.

Lembrando-se que a Estatística é uma teoria matemática que, trata da avaliação e do processamento de informações com incertezas associadas, é possível realizar análises estatísticas com o objetivo de quantificar a influência das incertezas no estudo da condição de estabilidade de estruturas em rocha, podendo ser determinados índices como a probabilidade de ruptura e o índice de confiabilidade da análise.
Uma das principais dificuldades em se unirem análises estatísticas com a modelagem numérica encontra-se na caracterização da condição de estabilidade da estrutura em rocha representada por um dado modelo. Em vários métodos analíticos, critérios de avaliação são fornecidos por meio de grandezas adimensionais, como o fator de segurança. Por outro lado, os modelos numéricos podem apresentar, como resultado, várias informações, como a distribuição de tensões e de deformações, deslocamentos e outras, as quais devem ser analisadas e comparadas pelo pesquisador para que seja emitido um juízo sobre a condição de estabilidade do modelo.

Portanto, com o objetivo de suprir essa dificuldade, bem como de automatizar o processo, é proposto um sistema de decisão para a verificação do estado do modelo numérico, indicando se ocorreu ou não a sua ruptura, possibilitando a aplicação das técnicas estatísticas ao método dos elementos distintos, as quais, normalmente, demandam a simulação de um grande número de alternativas (Cundall \& Hart, 1993).

Esse sistema é baseado na Lógica Paraconsistente, uma lógica não-clássica, criada, especialmente, para lidar com as situações de inconsistência e de indeterminação, que podem surgir quando são cruzadas várias informações, como as obtidas pelo processamento de um modelo numérico.

Essa metodologia será, então, aplicada a um caso de estudo da estabilidade de um talude de grandes dimensões, como ilustração de seu uso e abrangência em relação à análise determinística convencional.

\section{Metodologia 2.1 Confiabilidade estrutural}

Nas análises determinísticas convencionais, a avaliação da estabilidade de estruturas em rocha envolve incertezas decorrentes de muitos fatores, as quais, na maioria das vezes, não são levadas em conta no resultado obtido. A análise probabilística pode ser aplicada com o objetivo de quantificar as incertezas inerentes à condição de estabilidade, por meio da probabilidade de ruptura $(\alpha)$ e do índice de confiabilidade $(\beta)$, que exprime a confiabilidade da análise, ou do próprio fator de segurança, caso seja possível determiná-lo (Oberkampf et al., 2002).

As fontes de incerteza em estudos geotécnicos são:

- A variabilidade própria dos processos naturais, que se relaciona à variação natural encontrada no sistema físico sob consideração.

- A incerteza do modelo, refletindo a limitação dos modelos e métodos de análise adotados na representação do comportamento real do sistema em estudo.

- As incertezas dos parâmetros do modelo, provenientes da incapacidade de se quantificar, com precisão, os parâmetros de entrada do problema.

- As incertezas dos dados, incluindo erros de medição e de manipulação de dados, além da inconsistência de dados e sua não homogeneidade.

O objetivo da análise de estabilidade sob o enfoque probabilístico e da análise de risco é inferir a probabilidade de sucesso ou insucesso de uma estrutura e, portanto, a sua segurança, mediante o cálculo da probabilidade de ruptura ou da distribuição probabilística do fator de segurança. Trabalho esse que não é trivial, já que muitas incertezas estão presentes. Entre os métodos que tentam incorporar esse tipo de análise, destacam-se: o do "Segundo Momento de Primeira Ordem", o das "Estimativas Pontuais" e o "Método de Monte Carlo".

\subsection{Lógica Paraconsistente}

Como tratado na introdução desse trabalho, uma das principais dificuldades da modelagem numérica é a caracterização da condição de estabilidade do modelo estudado, pois esta tarefa é deixada a cargo do usuário. 
O processo de tomada de decisão executado por um pesquisador apresenta aspectos complexos inerentes à própria inteligência humana. Esse processo, entretanto, pode ser simulado de uma maneira adequada e implementado em um código computacional, de modo a fornecer respostas automáticas e rápidas.

Aspectos relativos à tomada de decisão envolvem a avaliação de informações que podem apresentar inconsistência, isto é, informações contraditórias como, por exemplo, algumas indicando "ruptura" e outras indicando "estabilidade", ou informações que apresentam indeterminação, ou seja, não indicando claramente um estado, tal como "ruptura" e "estabilidade". Essas informações, que podem ser quantitativas ou qualitativas, conjugadas a outros fatores tais como: tipo, finalidade e vida útil da estrutura modelada, podem ser levadas em conta na avaliação do modelo numérico analisado.

Portanto um sistema de análise automática de modelos numéricos faz-se necessário, para que se possa aplicar, por exemplo, o método de Monte Carlo e, assim, executar-se uma análise de confiabilidade estrutural, embasada estatisticamente. O sistema proposto é baseado na Lógica Paraconsistente, que é capaz de lidar com as diversas informações, muitas vezes controversas, provenientes do modelo numérico, constituindo um sistema com estrutura compacta, capaz de ajustes simples e coerentes com a realidade física, atuando de modo eficiente.

A Lógica Paraconsistente foi desenvolvida para desafiar o princípio da contradição. A lógica clássica, bem como outras lógicas não clássicas, não trata desse princípio. A Lógica Paraconsistente pode ser utilizada como a lógica subjacente de teorias inconsistentes e não triviais. Nela uma contradição não invalida (ou não trivializa) todo o sistema. Ela rompeu o paradigma caracterizado pela lógica de tradição aristotélica, possibilitando a aceitação da existência de teorias inconsistentes e a coexistência de estruturas lógicas incompatíveis entre si (Silva Filho \& Abe, 1997).
Sua modalidade anotada de anotação com dois valores, que será utilizada nesse trabalho, é uma classe de Lógica Paraconsistente, que trabalha com evidências e que admite contradição de um modo não trivial. Nesse sistema lógico, as anotações são representativas de graus de crença e de descrença atribuídos a uma dada proposição, dando-lhe conotações de valoração. Ela pode ser representada de modo particular, através de um reticulado de Hasse, conforme mostra a Figura 1, que associa valores lógicos a uma proposição.

Em tal reticulado, $(1,0)$ indica verdadeiro ou "crença total", $(0,1)$ indica falso ou "descrença total", $(1,1)$ indica inconsistência ou "crenças totalmente inconsistentes" e $(0,0)$ indica indeterminação ou "ausência total de crença".

Aplicando-se o raciocínio evidencial na Lógica Paraconsistente anotada proposta, dois valores são agora associados a uma anotação do reticulado, por isso esta será denominada de "lógica paraconsistente anotada de anotação com dois valores - LPA2v", com a associação a esse reticulado de variáveis proposicionais $\left(\mathrm{p}_{1}, \mathrm{p}_{2}, \mathrm{p}_{3}, \ldots, \mathrm{p}_{\mathrm{n}}\right)$, de constantes anotacionais e símbolos auxilia- res, e de conectivos lógicos: $\neg$ (negação), $\wedge$ (conjunção), $\vee($ disjunção $)$ e $\rightarrow$ (implicação).

O primeiro valor da anotação representa a evidência favorável à proposição $\mathrm{p}$ e o segundo valor da anotação representa a evidência contrária à proposição $\mathrm{p}$.

Será denominada de crença a evidência favorável à proposição $\mathrm{p}$, isto é, primeiro valor da anotação, e de descrença a evidência contrária à proposição $\mathrm{p}$, isto é, o segundo valor da anotação.

O grau de crença é simbolizado por $\mu_{1}$ e o grau de descrença, por $\mu_{2}$.

Com essas considerações, cada constante anotacional do reticulado é agora representada pelo par $\left(\mu_{1}, \mu_{2}\right)$, onde $\mu_{1}=$ grau de crença e $\mu_{2}=$ grau de descrença.

A idéia epistemológica intuitiva da associação de uma anotação $\left(\mu_{1}, \mu_{2}\right)$ a uma proposição $\mathrm{p}$ significa que o grau de crença em p é $\mu_{1}$, enquanto o grau de descrença é $\mu_{2}$. Por exemplo, intuitivamente, lê-se a fórmula $\mathrm{p}\left(\mu_{1}, \mu_{2}\right)$ como crêse em $\mathrm{p}$ com crença favorável até $\mu_{1} \mathrm{e}$ crença desfavorável até $\mu_{2}$ (Costa et al., 1999).

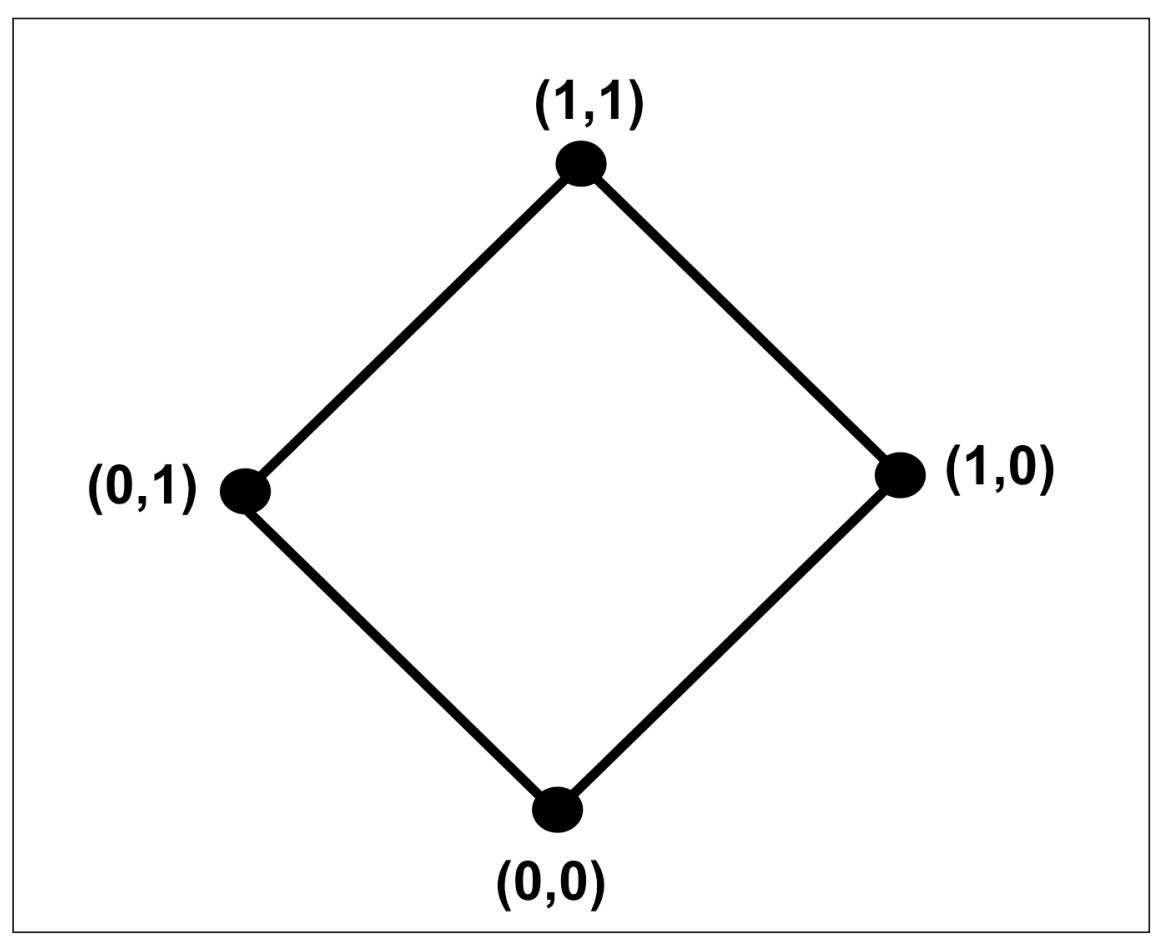

Figura 1 - Diagrama de Hasse - reticulado "quatro" (AKAMA e ABE, 1998). 


\section{Desenvolvimento do sistema}

A alimentação do sistema foi definida sobre dois canais de informação extraídos do modelo numérico, tal como necessário à aplicação da Lógica Paraconsistente anotada a dois valores. Esses canais de informação constituem-se em variáveis de monitoramento, que devem ser selecionadas pela relevância das informações nelas contidas (Bennaton, 1994).

Selecionadas as variáveis, é definida a "crença na ruptura", em cada um dos instantes de processamento de um modelo numérico, a partir da determinação de dois índices lógicos, que são funções das variáveis escolhidas: $\mu_{1}=$ grau de crença, obtido a partir do primeiro canal de informação, e $\mu_{2}=$ grau de descrença, obtido a partir do segundo canal de informação.

Esses índices serão utilizados para atribuir um valor lógico à proposição "Ocorre Ruptura do Modelo Numérico", segundo as categorias verdadeiro, falso, inconsistente ou indeterminado, sendo que os modelos numéricos classificados, segundo as duas últimas categorias, são desconsiderados, quando do cálculo da probabilidade de ruptura, pois, por sua própria definição, são modelos inviáveis, que não representam condições efetivamente possíveis de ocorrência prática.

\subsection{Escolha de variáveis}

Deve-se proceder à escolha apropriada das variáveis de monitoramento do modelo, a fim de que sejam calculados os índices $\mu_{1}$ e $\mu_{2}$. Em taludes de mineração de grandes dimensões, compostos de rocha muito fraturada ou bastante alterada, como o do caso estudado, o comportamento esperado está relacionado a deformações plásticas da grande massa de rocha solicitada pelo peso próprio. Nessas condições, o monitoramento da estabilidade do modelo numérico pode ser eficientemente realizado observando-se o "número de ele- mentos rompidos", elementos da malha de diferenças finitas, nos quais os blocos são discretizados e que sofreram ruptura, e o "deslocamento" sofrido por um ponto situado na região central da massa de rocha a sofrer escorrega-

Salienta-se que, para cada tipo de escavação, se em rocha bastante ou pouco fraturada, em superfície ou subterrânea, a grande ou pequena profundidade e de acordo com os métodos de lavra comumente utilizados na mineração, como, por exemplo, pilar e salão, desmonparâmetros de monitoramento podem ser escolhidos de modo diverso, de acordo com as características particulares do estudo.

\subsection{Definição do reticulado}

O desenvolvimento para a variação contínua de $\mu_{1}$ e $\mu_{2}$, no intervalo real $[0 ; 1]$, resulta em um reticulado paraconsistente difuso e, entre várias possibilidades, a mais simples (com resolução 2x2) é apresentada na Figura 2. mento. te por subníveis, corte e aterro etc., os

$\mathrm{O}$ reticulado da Figura 2 divide o espaço de $\mu_{1} \times \mu_{2}$ em 4 regiões distintas, que agrupam os resultados lógicos provenientes de uma simulação, conforme a Tabela 1, que também apresenta a decisão com respeito à condição de estabilidade do modelo numérico.

A definição das quatro regiões possibilita a classificação de condições de estabilidade, que não seriam facilmente enquadrados nas categorias simples de ruptura e não ruptura. Os comportamentos anômalos, decorrentes de inconsistência ou indeterminação, que ocorrem em processamentos, se tiverem que ser classificados apenas segundo as categorias binárias, poderão sobrecarregar a análise com informações incorretas sobre as condições de estabilidade dos modelos numéricos.

\section{Resultados}

Como exemplo de aplicação da metodologia proposta, foi analisado o talude final de uma mineração, cuja geometria é apresentada na Figura 3 (Rusilo, 2003).

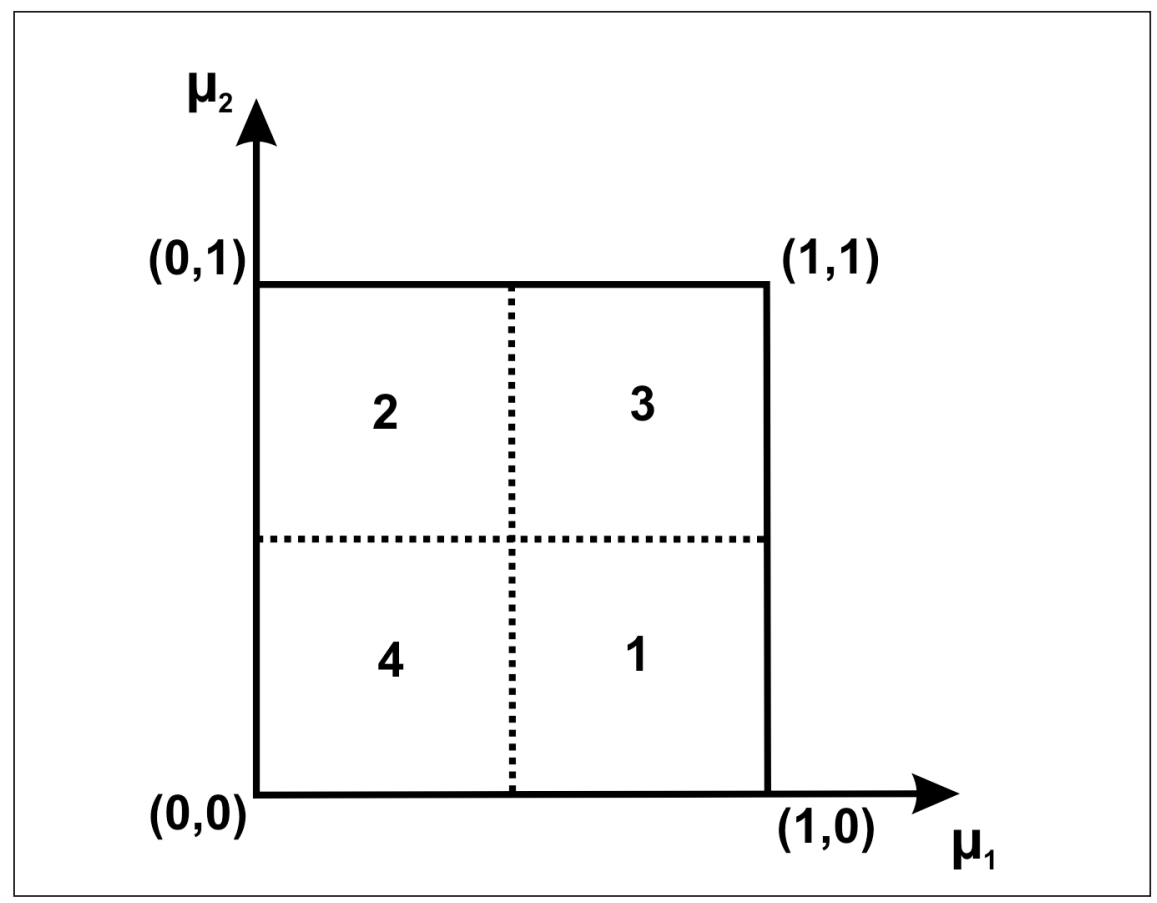

Figura 2 - Reticulado paraconsistente para análise de estabilidade do modelo numérico com resolução $2 \times 2$. 
Eduardo César Sansone et al.

Foram realizados 70 ensaios de compressão triaxial, 68 ensaios de compressão puntiforme, 14 ensaios de cisalhamento direto e 43 ensaios de compressão uniaxial com determinação do módulo de elasticidade e do coeficiente de Poisson no Instituto de Pesquisas Tecnológicas do Estado de São Paulo - IPT, e os parâmetros geomecânicos adotados para os materiais constituintes do talude são apresentados na Tabela 2.

Processando-se o modelo pelo método dos elementos distintos, obtêm-se vários tipos de informações, que indicam a estabilidade do modelo. Entretanto nada informam a respeito da condição em situações extremas, quando as incertezas e a variabilidade dos parâmetros adotados atuam desfavorecendo a estabilidade do modelo.

Não há um valor, como um fator de segurança global, ou uma probabilidade de ruptura, que seja associada ao modelo, para informar ao pesquisador o quão distante está o modelo da condição de ruptura.

Com a aplicação da metodologia proposta, foram combinados, na simulação pelo método de Monte Carlo, 1.384 valores amostrados a partir de distribuições de probabilidade normais representativas de cada um dos parâmetros, truncadas de modo a evitar a utilização de valores negativos. Foi constituído um hipercubo de 12 dimensões, correspondentes à variação dos parâmetros geomecânicos apresentados na Tabela 2, à exceção das densidades.

A combinação dos parâmetros produziu 1.384 modelos numéricos simulados, dos quais 462 não foram viáveis, isto é, não permitiram o processamento. Observa-se que são modelos numéricos com módulos de elasticidade bastante baixos, ainda que "possíveis" segundo a distribuição probabilística adotada, mas que apresentaram deformações superiores à ordem de grandeza do comprimento dos elementos utilizados na malha de diferenças finitas, na qual os blocos são discretizados no modelo, provocando a interrupção do algoritmo dos elementos distintos e impossibilitando seus processamentos.
Utilizando-se os dois índices $\mu_{1}=$ índice de elementos rompidos no modelo (grau de crença na ruptura) e $\mu_{2}=$ índice de deslocamento vertical do ponto $\mathrm{P}$ (grau de descrença na ruptura), foram monitorados o número de elementos rompidos no modelo e o deslocamento sofrido pelo ponto $\mathrm{P}$ (vide Figura 3).

O cálculo de $\mu_{1}$ e $\mu_{2}$ é realizado pela normalização dos valores dos parâmetros, entre os limites máximo e mínimo impostos para cada variável, e o cálculo dos limites $[0 ; 1]$, por meio das expressões 1 e 2 :

Tabela 1 - Divisão do espaço $\mu_{1} \times \mu_{2}$, na análise da ruptura

\begin{tabular}{c|c|c}
\hline Região & Resultado & Decisão \\
\hline 1 & 1 - Verdadeiro & Ruptura \\
\hline 2 & 0 - Falso & Não-Ruptura \\
\hline 3 & T - Inconsistente & Ruptura Não Característica \\
\hline 4 & $\perp-$ Indeterminado & Não-Ruptura Não Característica \\
\hline
\end{tabular}

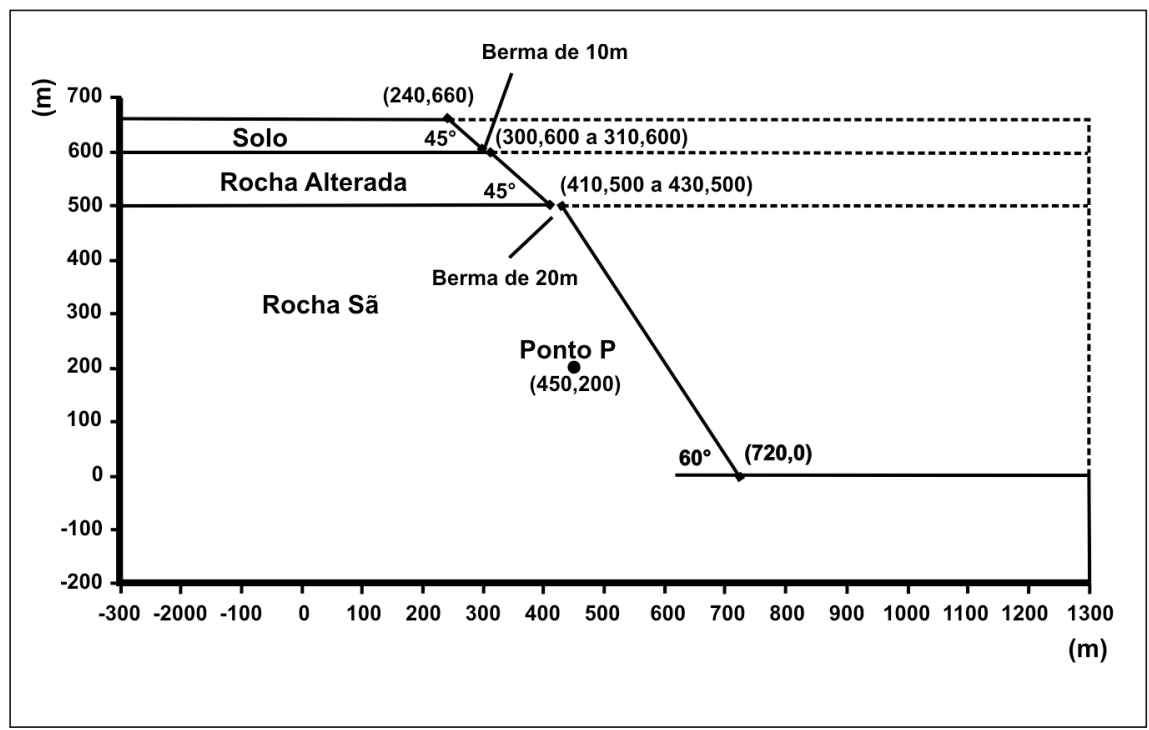

Figura 3 - Geometria do modelo numérico de talude final de cava de mineração.

Tabela 2 - Parâmetros adotados.

\begin{tabular}{c|c|c|c}
\hline Parâmetro & Rocha Sã & $\begin{array}{c}\text { Rocha } \\
\text { Alterada }\end{array}$ & Solo \\
\hline Densidade, $\rho$ & $2,674 \mathrm{t} / \mathrm{m}^{3}$ & $2,567 \mathrm{t} / \mathrm{m}^{3}$ & $2,200 \mathrm{t} / \mathrm{m}^{3}$ \\
\hline Coesão, $\mathrm{C}$ & $13,8 \mathrm{MPa}$ & $3,6 \mathrm{MPa}$ & $1,0 \mathrm{MPa}$ \\
\hline Ângulo de Atrito Interno, $\phi$ & $70,2^{\circ}$ & $50,0^{\circ}$ & $28,0^{\circ}$ \\
\hline Módulo de Elasticidade, $\mathrm{E}$ & $118.217 \mathrm{MPa}$ & $32.903 \mathrm{MPa}$ & $31.683 \mathrm{MPa}$ \\
\hline Coeficiente de Poisson, $v$ & 0,32 & 0,25 & 0,25 \\
\hline
\end{tabular}


$\mu_{1}=\frac{V_{1}}{\left(V_{1 \text { máx }}-V_{1 \text { min }}\right)}-\frac{V_{1 \text { min }}}{\left(V_{1 \text { máx }}-V_{1 \text { min }}\right)}$

$\mu_{2}=\frac{-\mathrm{V}_{2}}{\left(\mathrm{~V}_{\text {máx }}-\mathrm{V}_{2 \text { min }}\right)}+\frac{\mathrm{V}_{2 \text { máx }}}{\left(\mathrm{V}_{\text {máx }}-\mathrm{V}_{2 \text { min }}\right)}$

Onde: $\mathrm{V}_{1}$ e $\mathrm{V}_{2}=$ valores das variáveis 1 e 2 monitoradas; $\mathrm{V}_{1 \text { máx }}$ e $\mathrm{V}_{2 \text { máx }}=$ valores máximos das variáveis monitoradas; $\mathrm{V}_{1 \text { min }} \mathrm{e}_{2 \text { min }}=$ valores mínimos das variáveis monitoradas.

Os valores obtidos dos processamentos foram normalizados pelas expressões anteriormente apresentadas, e plotados no reticulado decisório. O reticulado utilizado para a análise foi de ordem $3 \times 3$, como pode ser visto na Figura 4. O reticulado passa a apresentar contornos mais complexos que um $2 \times 2$, embora não arbitrários, pois devem ser definidos pelo pesquisador com base na agregação de dados da experiência.

A Figura 5 apresenta os resultados analisados pelo reticulado.

Os valores de corte são escolhidos dois a dois. Para a porcentagem de elementos rompidos, o valor de corte inferior corresponde a $35,0 \%$ e o superior, a $40,0 \%$. O valor de corte inferior de deslocamento corresponde a $3,0 \mathrm{~cm}$ e o superior, a $5,0 \mathrm{~cm}$. O número total de modelos numéricos não rompidos foi de 666 e foram verificados 27 modelos numéricos com ruptura e os que apresentaram situação indeterminada ou inconsistente somaram 229, representando $24,8 \%$ do total (Tabela 3).

Para o cálculo da probabilidade de ruptura, foram considerados apenas os modelos numéricos rompidos e não rompidos, descartando-se os demais modelos inviáveis, o que perfaz 693 modelos numéricos, dos quais 27 apresentaram-se rompidos. Esses valores fornecem a probabilidade de ruptura $\alpha$ de 3,9\%. Esse valor e o correspondente índice de confiabilidade $\beta$ de $96,1 \%$ ( $\beta=1-\alpha$ ) podem ser considerados aceitáveis para a estabilidade de um talude de mineração, levando-se em conta que o local é de acesso restrito apenas a funcionários treinados.

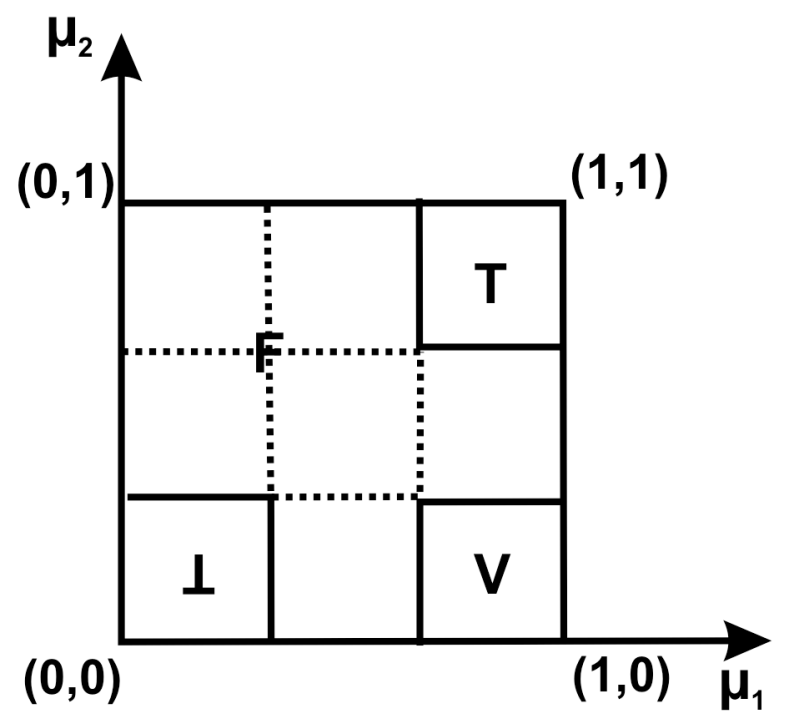

Figura 4 - Reticulado decisório para a Lógica Paraconsistente com resolução 3×3.

\section{Conclusões}

O método de análise probabilística de Monte Carlo pode ser utilizado em associação ao método dos elementos distintos, desde que seja implementado um sistema que automatize a classificação da condição de estabilidade dos vários modelos numéricos processados. Assim, foi possível o desenvolvimento conceitual de uma metodologia para análises probabilísticas no estudo da condição de estabilidade de estruturas em rocha, com a aplicação de modelagem numérica.

Aplicando-se a metodologia desenvolvida, verificou-se que ela apresenta resultados realísticos, com notável aumento das informações obtidas em relação ao estudo de estabilidade determinístico, tradicionalmente executado, obtendo-se com ela a probabilidade de ruptura de um talude final de cava de mina, a partir da análise dos resultados das duas variáveis monitoradas no modelo numérico.

Em simulações numéricas conjugadas à aplicação do método de Monte Carlo, a quantidade de modelos processados, e que devem ser analisados, pode exceder a capacidade humana ou, pelo menos, impede que o estudo possa ser concluído em um prazo razoável. A automatização necessária foi obtida mediante a aplicação da Lógica Paraconsistente, que alia simplicidade à sua sistematização formal, apresentando-se como uma ferramenta útil no desenvolvimento de uma metodologia de baixa complexidade e de aplicação geral, podendo ser aplicada a métodos numéricos diversos, objetivando a solução de uma questão prática de engenharia.

\section{Referências bibliográficas}

AKAMA, S., ABE, J. M. Constructive logics as annotated logics. São Paulo: IEA-USP, 1998.

BENNATON, J. F. Modos de pensar e sistemas de controle - um ponto de vista na história. São Paulo: IEA-USP, 1994. 


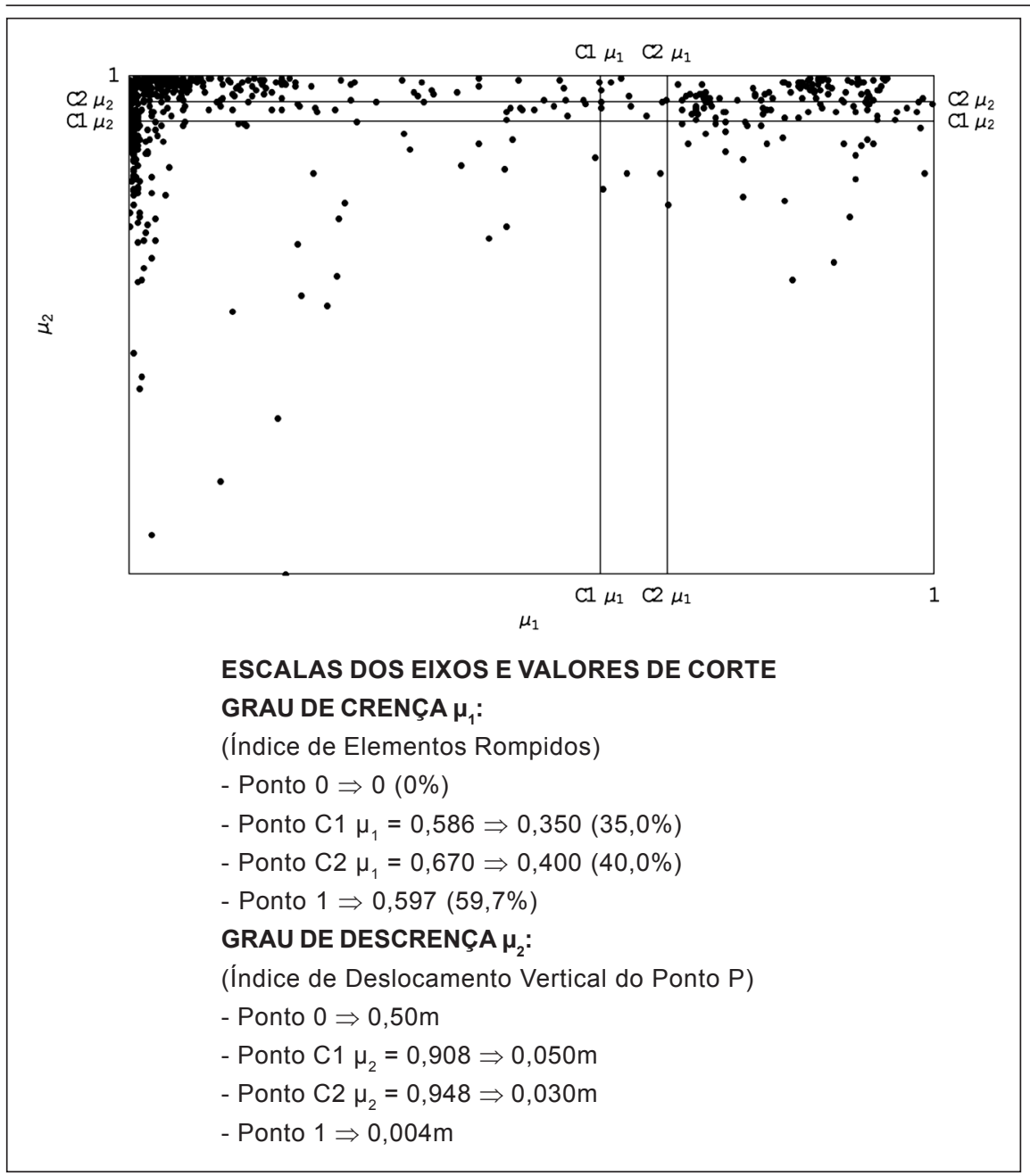

Figura 5 - Aplicação da Lógica Paraconsistente em um reticulado decisório com resolução $3 \times 3$ com valores de corte impostos pelo pesquisador.

Tabela 3 - Número de modelos numéricos classificados em cada estado.

\begin{tabular}{c|c|c}
\hline \multirow{2}{*}{ Estado } & \multicolumn{2}{|c}{ Modelos Processados } \\
\cline { 2 - 3 } & Número total & $\%$ \\
\hline Não Ocorre Ruptura & 666 & 72,2 \\
\hline Ocorre Ruptura & 27 & 2,9 \\
\hline Condição Indeterminada & 146 & 15,8 \\
\hline Condição Inconsistente & 83 & 9 \\
\hline Total & 922 & 100 \\
\hline
\end{tabular}

COSTA, N. C. A. et al. Lógica paraconsistente aplicada. São Paulo: Atlas, 1999.

CUNDALL, P. A., HART, R. D. Numerical modeling of discontinua. In: HUDSON, J. A. et al. Comprehensive rock engineering: principles, pratice and projects. Oxford: Pergamon Press, 1993. v.2, cap.9: 231-44.

DEKHTYAR, A., SUBRAHMANIAN, V. S. Hybrid probabilistic programs. The Journal of Logic Programming. Oxford: 2000. v.43, n.3.

GLASER, S. D, DOOLI, D. M. New directions in rock mechanics. Report on a forum sponsored by the American Rock Mechanics Association. International Journal of Rock Mechanics and Mining Sciences. Oxford, 2000. v. 37.

HOEK, E. Rock engineering. The Application of modern techniques to underground design. São Paulo: ABMS, 1998.

JING, L. A review of techniques, advances and outstanding issues in numerical modelling for rock mechanics and rock engineering. International Journal of Rock Mechanics \& Mining Sciences, 40. 2003.

OBERKAMPF, W.L., DELAND, S.M., RUTHERFORD, B.M., DIEGERT, K.V., ALVIN, K. F. Error and uncertainty in modeling and simulation. Reliability Engineering \& System Safety. Oxford, 2002. v.75, n.3.

RUSILO, L. C. Aplicação da lógica paraconsistente à análise da estabilidade de estruturas em rocha. São Paulo: Departamento de Engenharia de Minas da Escola Politécnica da USP, 2003. ( Tese de Doutorado).

SANSONE, E. C. Estudo de aspectos geomecânicos aplicados ao projeto de minas subterrâneas. São Paulo: Departamento de Engenharia de Minas da Escola Politécnica da USP, 1997. (Dissertação de Mestrado).

SILVA FILHO, J. I. DA., ABE. J. M. Módulos analisador paraconsistente: uma proposta de circuito lógico paraconsistente. São Paulo: IEA-USP, 1997.

YOSHIKAWA, N. K. Nova metodologia de avaliação de estabilidade de encostas rochosas. São Paulo: Departamento de Engenharia de Minas da Escola Politécnica da USP, 1997. (Tese de Doutorado).

\section{Artigo recebido em 29/02/2008 e} aprovado em $03 / 12 / 2008$. 